\title{
Treatment Decisions for Advanced Non-Squamous Non-Small Cell Lung Cancer: Patient and Physician Perspectives on Maintenance Therapy
}

\author{
Suzanne McMullen ${ }^{1} \cdot$ Lisa M. Hess ${ }^{2} \cdot$ Edward S. Kim $^{3} \cdot$ Benjamin Levy $^{4} \cdot$ Mohamed Mohamed $^{5} \cdot$ David Waterhouse $^{6}$. \\ Antoinette Wozniak $^{7} \cdot$ Sarah Goring $^{1} \cdot$ Kerstin Müller $^{1}$ (1) $\cdot$ Catherine Muehlenbein $^{2} \cdot$ Himani Aggarwal $^{2} \cdot$ Yajun Zhu $^{2}$. \\ Ana B. Oton ${ }^{2} \cdot$ Jennifer L. Ersek ${ }^{3} \cdot$ Katherine B. Winfree ${ }^{2}$
}

Published online: 21 August 2018

(c) The Author(s) 2018

\begin{abstract}
Introduction Advanced non-small cell lung cancer (NSCLC) is a severe disease with burdensome symptoms and traditionally poor outcomes. The treatment of advance disease is based on chemotherapy, with the recent addition of immunotherapy. Patients who respond to initial treatment can opt to receive maintenance therapy (MT). It is important to understand why patients with advanced NSCLC choose to accept or refuse therapy, and how physician recommendations play into this decision-making process. This study characterized patient and physician decision-making regarding treatment for patients with advanced non-squamous NSCLC in the USA using the example of MT.

Methods and Materials This study employed multiple approaches: patient interviews, a patient survey, and a physician survey. Qualitative interviews were conducted among patients who had been offered MT to identify factors influencing treatment decision-making. The patient survey explored the decision-making process and quantified challenges and motivators for receiving MT. The physician survey included a discrete choice experiment to understand the relationship between physician treatment recommendations and patient characteristics.

Results Interviewed patients $(n=10)$ were motivated to receive MT in the hope of extending their lives and being proactive against their cancer, and they anticipated reduced adverse effects compared with first-line therapy. Surveyed patients $(n=77)$ described several deterrents to receiving therapy; the most prominent was severity of adverse effects, which was an influencing factor for $34 \%$ of patients. The major motivator for receiving therapy was the potential to extend life, which influenced $97 \%$ of patients. A total of 100 oncologists participated in the physician survey. Patients' lack of treatment motivation/inconvenience, disease progression, presence of severe renal co-morbidities, and older age decreased the likelihood of physicians recommending the use of MT.

Conclusion This study identified challenges and motivators influencing advanced NSCLC patients' decisions to accept or refuse therapy, as well as patient and disease characteristics associated with physician's treatment recommendations for MT.
\end{abstract}

Electronic supplementary material The online version of this article (https://doi.org/10.1007/s40271-018-0327-3) contains supplementary material, which is available to authorized users.

Kerstin Müller

Kerstin.Mueller@iconplc.com

1 ICON Plc, Vancouver, BC, Canada

2 Eli Lilly and Company, Indianapolis, IN, USA

3 Levine Cancer Institute, Atrium Health, Charlotte, NC, USA

4 Mount Sinai Health Systems, New York, NY, USA

5 Cone Health, Greensboro, NC, USA

6 Oncology Hematology Care (OHC), Cincinnati, OH, USA

7 Karmanos Cancer Institute, Detroit, MI, USA 


\section{Key Points for Decision Makers}

Patients were knowledgeable about goals and outcomes of maintenance therapy for non-small cell lung cancer (NSCLC).

Decision-making regarding treatment of NSCLC is a shared process between patients and physicians; physicians were the main source of information about therapy options and were almost always strongly involved in the decision-making process.

A main motivator for patients to accept therapy is the potential to extend survival.

A major challenge to receiving therapy is the possibility of adverse effects.

Discussions between patients and physicians on goals of therapy and management of possible adverse effects play an important role in patients' decision to receive therapy.

\section{Introduction}

Lung cancer is a leading cause of cancer mortality in the USA, accounting for approximately $27 \%$ of cancer deaths [1]. Non-small cell lung cancer (NSCLC) is responsible for the majority of lung cancer cases and is associated with poor outcomes. Five-year survival rates for NSCLC vary considerably by stage, from 56 to $5 \%$ for those diagnosed in the localized stage I versus IV [1]. However, only $16 \%$ of lung cancers are diagnosed at a localized stage [1], while $40 \%$ of NSCLC patients are diagnosed with stage IV disease.

NSCLC is usually associated with burdensome symptoms that negatively impact patients' quality of life $[2,3]$. These can include pain, coughing, shortness of breath, hemoptysis, fatigue, and loss of appetite [3].

Advanced NSCLC is usually treated systemically with chemotherapy, targeted therapy based on specific mutation status, immunotherapy, or a combination of these [7], and is often followed by maintenance therapy (MT) for patients with stable or responding disease.

It is important to understand patient decisions regarding advanced NSCLC therapy, and to identify barriers to, and motivators for, the acceptance of therapy from the patient perspective. It is also relevant to understand the role of the physician in this decision, and physicians' perspectives regarding recommending specific treatments to individual patients. An improved understanding of these topics may assist in the provision of patient-centered care.

The objective of this study was to identify factors that influence patient and physician decision-making regarding treatment of advanced non-squamous NSCLC, using the example of MT among patients eligible for MT according to the National Comprehensive Cancer Network (NCCN) guidelines. At the time this study was initiated in 2015/2016, MT was the mainstay therapy for advanced NSCLC patients following first-line treatment. MT typically involves the continuation of one or more of the treatments received as firstline (continuation MT), but may involve a switch to a new agent not administered during first-line treatment (switch MT). Clinical trials have demonstrated progression-free survival (PFS) and overall survival (OS) benefits following both continuation and switch MT [4-6], but as with many treatment approaches, these advantages are weighed against potential adverse effects, impact on quality of life, and inconveniences that may be associated with active treatment.

\section{Methods}

Patient qualitative interviews, a paper-based patient survey, and an online physician survey were conducted to elicit and quantify the factors associated with treatment decision-making in advanced NSCLC.

\subsection{Patient Interviews and Patient Survey}

The NCCN guidelines at the time this study was initiated in 2015 recommended the use of MT among patients with non-squamous NSCLC without actionable mutations, with an Eastern Cooperative Oncology Group (ECOG) performance status (PS) of $\leq 2$, and without disease progression [7]. Eligible patients for both the interviews and survey thus included US adults with advanced non-squamous NSCLC who completed four or more cycles of first-line platinumbased chemotherapy without progression. Patients with an epidermal growth factor receptor $(E G F R)$ mutation or an anaplastic lymphoma kinase (ALK) translocation, or who participated in a clinical trial within 30 days of screening, were excluded. Participants were enrolled from one clinical site for the qualitative interviews (North Carolina) and from five clinical sites for the survey (North Carolina [two sites], Ohio, Michigan, and New Jersey). Participants received compensation for their time. The Western Institutional Review Board (WIRB) approved study procedures for the sites in Ohio, Michigan, and New Jersey. The Cone Health Institutional Review Board (IRB) and Chesapeake IRB approved study procedures for the two sites in North Carolina. Study participants provided informed consent prior to data collection.

The in-person qualitative interviews followed a semistructured interview guide (see Electronic Supplementary Material Appendix 'Patient Interview Guide') designed to elicit concepts associated with treatment decision-making. 
Interviews were conducted between June and August 2015 and lasted from 30 to $60 \mathrm{~min}$. Additional interviews were scheduled until information saturation was achieved (i.e., the point at which no new concepts emerged). Qualitative interviews were analyzed through thematic analysis [8], using MaxQDA software (VERBI Software GmbH, Berlin, Germany). A codebook was developed based on the interview guide and initial review of the transcripts. These predetermined codes were assigned by one coder to sections of transcripts, and new codes were added and the code list refined to cover all relevant information captured. Codes were then grouped into concepts, defined by the content of quotation coded and supported by example quotations.

The concepts identified in the qualitative interviews informed the content for the quantitative cross-sectional patient survey, which was conducted between April and September 2016. The survey contained questions on patients' demographic and clinical information, treatment decisionmaking process, treatment experience, and challenges and motivators to receiving treatment. Patients were asked if each challenge and motivator was present for them and how influential it was on their decision. The survey was piloted among ten participants to ensure internal validity. The target sample size for the survey was 100 patients. The administration of the patient survey was initially planned to be inperson, but was amended during data collection to allow the survey to be mailed to participants to increase enrollment.

The survey data were analyzed using descriptive statistics using SAS $^{\circledR}$ version 9.4 (SAS Institute, Cary, NC, USA).

\subsection{Physician Survey}

An online cross-sectional physician survey was conducted in mid-2015 to evaluate practice patterns and decision-making processes for MT recommendations. The survey is available in the Electronic Supplementary Material (Appendix 'Physician Survey'). Eligible physicians were oncologists who specialized in lung cancer and were treating at least ten lung cancer patients, including at least one advanced non-squamous NSCLC patient, at the time of study entry, and who prescribed chemotherapy to patients with advanced NSCLC. The target sample size was 100 physicians, recruited from a research database (Medefield Ltd, New York, NY, USA). Physicians were recruited to ensure an equal distribution of participants practicing in an academic or hospital and a community-based setting. Participants received compensation for their time. The WIRB approved study procedures.

The survey included a series of questions regarding physicians' clinical experience and practice setting, as well as a discrete choice experiment (DCE) that presented physicians with hypothetical advanced-stage NSCLC patient profiles and asked if they would recommend switch MT, continuous MT, or no MT. The patient profiles were defined by six attributes, each with four levels. The attributes and levels included Response to first-line treatment (levels: complete response, partial response, stable disease, progression); First-line treatment tolerability (levels: no adverse events [AEs], one grade 1-2 AE, two grade 1-2 AEs, one grade 3-4 AE); Age (levels: 45, 58, 68, 80); Co-morbidities (levels: no active co-morbidities, severe renal impairment, mild renal impairment, one serious co-morbidity [hepatitis B, diabetes, chronic obstructive pulmonary disease, cerebrovascular disease, or cardiovascular disease] with the individual serious co-morbidities presented in equal proportion across profiles); Patient motivation and convenience of treatment (levels: motivated and convenient, motivated but not convenient, not motivated but convenient, not motivated and not convenient); and Insurance status (levels: 0\%, 5\%, 10\%, $20 \%$ co-pay). A fractional factorial design was employed, with 12 orthogonal and balanced profiles presented to each physician. The DCE profiles were designed using Sawtooth software (Sawtooth Software, Inc., Provo, UT, USA). Physicians were asked to describe their alternative management strategy if they chose not to recommend MT. More information on the DCE design process can be found in the Electronic Supplementary Material.

The DCE responses were analyzed with multiple logistic regression models, using $\mathrm{R}$ version 3.3.3. Random effects were included to account for the fact that each physician was answering multiple questions and may have systematic preferences.

\section{Results}

\subsection{Patient Interviews}

Ten qualitative interviews were conducted among advanced NSCLC patients to understand their treatment decision experience. After the tenth interview, information saturation was reached. Four of the interviewees were male; the mean age was 64 years. Interviewees provided individual perspectives of their involvement in treatment decision-making. Table 1 lists the main concepts related to decision-making with regards to treatment that were identified in the interviews, and presents quotes from patients relating to each concept. Some patients described being presented treatment options, with their physician making a recommendation for their consideration, whereas others felt that the treatment path was their physician's decision. Patients described discussions with the physicians as focusing on the expected treatment experience. Patients appreciated their physician's use of lay language over technical terms, and valued the support that they received from their family and friends when making a treatment decision. Interviewed patients understood that MT had the goal of preventing or slowing tumor growth 
Table 1 Concepts relating to treatment decision-making identified in the patient interviews, with patient quotes

Concept

Reduce/stabilize tumor size

Efficacy in general

HCP recommendation

HCP discussion

Adverse effects

Advance research

Dosing/regimen

Discussion with family or friends
Patient quotes

"[T]here's a possibility that it could help shrink or keep it under control what was there showing at that time." [101-010]

"Well, number one it was, um, the ability that hopefully the more, ah, treatment or medicine I got, it would help more-it would shrink the tumor more." [101-007]

"Dr. - explained to me, you know, that, um, if I would go on maintenance it would be better as far as preventing it from growing, and each time I have had scans, every three months, and it has not grown." [101-006]

"Just the fact that it was-it could make it better." [101-007]

"[I]t has been already been researched to be, um, effective, um, with that progression, with that point of progression, and to me that says okay, it is-it is an okay drug." [101-005]

"One obviously would be the effectiveness, how effective it is going to be compared to what I'm doing now. If somebody came up today and said okay, I've got this, um, ah, drug A and we want you to go to this instead of what you're doing now, obviously the first thing I'd want to know is how effective it is." [101-002]

"Well, there really wasn't any. I mean, he just said that, uh, you know, my cancer was decreasing, so it was now time to put me on maintenance. That was pretty much all that was really said. I never questioned it, so. I mean, I trusted his judgment." [101-009]

"[T]here isn't another course of treatment. I didn't have choices. I didn't-it wasn't do you want A, B or C. It was kind of like this is what we have... This is the course of treatment for what you've got." [101-001]

"[J]just shortly before I finished up my chemo treatments, um, he said that he might want me to, ah, consider it and he explained it all to me about the maintenance drug." [101-010]

"They told us what it would involve, you know, the time-time, ah, it takes and then the effort it would take for my body and, um, you know, how weekly, um, that works and biweekly and whenever I go to maintenance. The whole thing is laid out at the beginning to let you know." [101-002]

"But—but, ah, he explained that to me. He says you're going to feel some effects of chemo just like, you know, like you did from the regular chemo, but it's not going to be as severe and it's not going to be as long. And what he told me is true. So I-I - I have no regrets. He didn't-he didn't force me to do it. He explained the pros and cons and, ah, as far - like I told you, as far as I was concerned, it was a no brainer." [101-004]

"Mainly what were the side effects of the drug, the long-term and well, the short term and-and the long term side effects. Um, I did some research and talked with someone at [NAME] and, um, and, um, from that, ah, that was one of the things that helped-helped me make the decision to go ahead." [101-005]

"[Doctor] says you're going to feel some effects of chemo just like, you know, like you did from the regular chemo, but it's not going to be as severe and it's not going to be as long." [101-004]

"As far as the side effects, they give you-they gave us a big, um, manual to read and-and tells you all the side effects that's possible that could happen." [101-002]

"II]t helps me to feel safe doing the, ah, um, study program and being on the maintenance chemo and hoping in the meantime that it helps the research." [101-002]

"[T]his was also a factor, which I wasn't taking as much medicine which had cut back on it and plus I wasn't taking it as often which I said it was just like every two weeks and then I had a rest period." [101-007]

"Did I have questions? Um, probably you mean I don't have to come but every three weeks? And the doses will be smaller and take less time?" [101-006]

"But the regular maintenance it was the inconvenience, the going to the-going to the clinic every week, getting blood drawn, and then after getting the chemo, I was-I was pretty sick for, you know, three or four, five days. But the maintenance chemo, I feel actually a little worn out a couple of days, but then it just day by day by day I just feel, you know, stronger and not as tired. Um, the regular chemo compared to the maintenance chemo is nothing." [101-004]

"The only one, my wife was with me all the time that this was going on and, ah, ah, we discussed it, do I or don't I take it, and, ah, she was with me 100 percent. Let's try it and see. We don't have nothing to lose." [101-010]

"Well, I learned a little bit from my son even though -I mean, he had the same diagnosis. But he reacted differently and he had it in different places than I do, so." [101-009]

"[Doctor] told me I could take time to, you know, decide what I wanted to do, which I took about I guess a couple of days and, you know, through the process of discussion with my family and everything, I decided I was still-I would take the maintenance treatment." [101-007] 
Table 1 (continued)

Concept
Patient quotes
"And I feel like if I'm not doing at least the maintenance I don't feel like I'm doing all I can do to-to battle
my cancer... You're still going to be doing something and participating in your treatment. And that's-
that's what I want. I want to feel-I want to feel like I'm proactive. I want to feel like I'm doing everything I
can to fight this." [101-011]
“And on the-on the flip side also is to know that it is working and so there is no, um, um, you know, doubt
that the cancer might come back because unless you scan every six weeks and it shows that it is not coming
back, it helps me to feel safe doing the, ah, um, study program and being on the maintenance chemo." [101-
002]
"I mean, I hadn't taken as much drugs and plus I hadn't take it as often, you know, during the month, plus,
ah, you know, just being, ah, wanted to be proactive. I wanted to do as much as I can, you know, to-to, ah,
get the situation where, um, I feel good about myself." [101-007]

$H C P$ healthcare professional

and subsequently identified this goal as a key motivator for receiving treatment. Several patients mentioned that it was important for them to feel that they were taking active steps to treat the cancer. Patients recognized adverse effects as a barrier to MT, but some felt that the anticipated improvement from first-line adverse effects was also a motivator. The treatment regimen was noted as an inconvenience by one patient. The barriers and motivators identified in the interviews were used to populate the survey.

\subsection{Patient Survey}

Seventy-seven patients were eligible and participated in the patient survey. The mean age was 65.4 years at diagnosis and 67.5 years at the time of the survey. Just over half of the patients $(55 \%)$ were female, the majority $(73 \%)$ were Caucasian, and nearly half (49\%) were married or cohabitating. Only $42 \%$ of patients reported complete insurance coverage, while $44 \%$ reported partial coverage. All but one patient had selected to receive MT. There were no patients who were eligible to participate but had yet to make a decision about MT.

Nearly all patients $(96 \%, n=74)$ reported learning about MT from their oncologist (Table 2). Other sources of information for the majority of patients included nurses and materials provided by healthcare providers. The majority of patients $(87 \%, n=67)$ reported multiple MTrelated discussions with their oncologist, beginning most frequently prior to first-line treatment $(44 \%, n=34)$, when the treatment plan and options were explained. Others reported that the initial discussions began at the beginning of or during first-line treatment $(17 \%)$ or after first-line treatment $(23 \%)$. Most patients indicated that they completely understood the goals of MT $(70 \%, n=54)$. Over $80 \%$ of patients reported that MT could prevent cancer from growing, may shrink the existing tumor, and may extend their life. While $55 \%$ of patients $(n=42)$ acknowledged that MT would not provide a cure, $25 \%(n=19)$ believed cure was possible. The majority $(77 \%, n=59)$ reported anticipating an improvement in quality of life compared with first-line therapy.

The majority of patients $(90 \%, n=69)$ felt that they had a choice of whether or not to receive MT after talking to their oncologist, whereas $8 \%$ did not perceive this treatment path as a choice. This aligns with the findings from the qualitative interviews, in which patients indicated that they felt the decision had been made for them by the oncologist. Nearly all patients $(95 \%, n=73)$ reported involvement of their oncologist in the decision. For $42 \%(n=32)$, the decision involved a spouse and for $43 \%(n=33)$, the decision involved other family members.

Based on a pre-specified list generated from the qualitative interviews, the most frequently reported challenge regarding the decision to accept therapy was the anticipated severity of potential adverse effects $(34 \%, n=26)$, followed by the anticipated impact on quality of life $(26 \%$, $n=20$; Table 3 ). Among patients who perceived adverse effects to be a challenge, fatigue and nausea/vomiting were the primary concerns $(60 \%, n=15$ and $56 \%, n=14$ of patients, respectively). However, only some patients who reported a specific adverse effect as a challenge had experienced the adverse effect of concern during first-line therapy (ranging from 0 to $40 \%$ ). For patients reporting challenges, $12-36 \%$ felt that the challenge was very influential to their decision, depending on the specific challenge.

All pre-identified motivators were influential to the decision-making for the majority of surveyed patients, ranging from $58 \%$ who reported fear of death as an influential motivator to $97 \%$ who reported ability to extend life as an influential motivator (Table 3 ). The one patient who declined MT reported that all pre-identified items were motivators except fear of death. For patients reporting motivators, the majority (ranging from 70 to $86 \%$ ) felt that the motivator was very influential to their decision, depending on the specific motivator. 
Table 2 Information sources and decision-making among all non-small cell lung cancer survey participants and stratified by treatment decision status

\begin{tabular}{|c|c|c|c|c|c|c|}
\hline \multirow[t]{2}{*}{ Information sources and decision-making factors } & \multicolumn{2}{|c|}{$\begin{array}{l}\text { All partici- } \\
\text { pants }(n=77)\end{array}$} & \multicolumn{2}{|c|}{$\begin{array}{l}\text { Chose MT } \\
(n=76)\end{array}$} & \multicolumn{2}{|c|}{$\begin{array}{l}\text { Decided } \\
\text { against MT } \\
(n=1)\end{array}$} \\
\hline & $n$ & $\%$ & $n$ & $\%$ & $n$ & $\%$ \\
\hline \multicolumn{7}{|l|}{ Sources used to learn about maintenance therapy ${ }^{\mathrm{a}}$} \\
\hline Treating oncologist & 74 & 96.1 & 73 & 96.1 & 1 & 100.0 \\
\hline Nurse & 49 & 63.6 & 49 & 64.5 & 0 & 0.0 \\
\hline Materials provided by healthcare professionals & 43 & 55.8 & 43 & 56.6 & 0 & 0.0 \\
\hline Spouse/partner & 13 & 16.9 & 13 & 17.1 & 0 & 0.0 \\
\hline Children/family members (other than spouse/partner) & 23 & 29.9 & 23 & 30.3 & 0 & 0.0 \\
\hline Friends & 18 & 23.4 & 18 & 23.7 & 0 & 0.0 \\
\hline Online & 30 & 39.0 & 30 & 39.5 & 0 & 0.0 \\
\hline Media (TV, magazines, news) & 16 & 20.8 & 16 & 21.1 & 0 & 0.0 \\
\hline No sources used & 3 & 3.9 & 3 & 4.0 & 0 & 0.0 \\
\hline \multicolumn{7}{|l|}{ Timing of first discussion about MT with treating oncologist } \\
\hline Prior to any treatment & 34 & 44.2 & 33 & 43.4 & 1 & 100.0 \\
\hline At beginning of first-line chemotherapy & 7 & 9.1 & 7 & 9.2 & 0 & 0.0 \\
\hline During first-line chemotherapy & 6 & 7.8 & 6 & 7.9 & 0 & 0.0 \\
\hline After first-line chemotherapy & 18 & 23.4 & 18 & 23.7 & 0 & 0.0 \\
\hline Other & 4 & 5.2 & 4 & 5.3 & 0 & 0.0 \\
\hline \multicolumn{7}{|l|}{ Number of discussions of MT with oncologist } \\
\hline 1 & 6 & 7.8 & 6 & 7.9 & 0 & 0.0 \\
\hline $1+$ & 67 & 87.0 & 66 & 86.8 & 1 & 100.0 \\
\hline Does not remember & 4 & 5.2 & 4 & 5.3 & 0 & 0.0 \\
\hline \multicolumn{7}{|c|}{ Felt they had a choice on whether or not to start MT after talking to oncologist about MT } \\
\hline Yes & 69 & 89.6 & 68 & 89.5 & 1 & 100.0 \\
\hline No & 6 & 7.8 & 6 & 7.9 & 0 & 0.0 \\
\hline Does not remember & 2 & 2.6 & 2 & 2.6 & 0 & 0.0 \\
\hline \multicolumn{7}{|l|}{ Involvement in decision regarding maintenance therapy ${ }^{a}$} \\
\hline Treating oncologist & 73 & 94.8 & 72 & 94.7 & 1 & 100.0 \\
\hline Spouse/partner & 32 & 41.6 & 31 & 40.8 & 1 & 100.0 \\
\hline Children/family members (other than spouse/partner) & 33 & 42.9 & 33 & 43.4 & 0 & 0.0 \\
\hline Other & 4 & 5.2 & 4 & 5.3 & 0 & 0.0 \\
\hline Nobody, made decision on their own & 7 & 9.1 & 7 & 9.2 & 0 & 0.0 \\
\hline
\end{tabular}

$M T$ maintenance therapy, $T V$ television

${ }^{a}$ Can add up to more than $100 \%$ because multiple options could be selected

\subsection{Physician Survey}

One hundred physicians completed the survey; $81 \%$ were male, with a mean of 15.4 years of experience post-residency. Physicians practiced in the eastern (28\%), mid-west $(24 \%)$, southern $(27 \%)$, and western $(21 \%)$ regions of the USA, and cared for a median of 50 NSCLC patients (range: $8-800$ ). Physicians reported using MT, on average, among $61 \%$ of patients with advanced non-squamous NSCLC, while seven physicians $(7 \%)$ reported never prescribing MT among this population. Physicians also reported using MT among patients not included in the NCCN guideline recommendations, such as those with advanced non-squamous NSCLC that were stable following targeted therapy
(65\%) and following second-line chemotherapy (58\%), patients with stage IIIa disease (38\%), and patients with squamous NSCLC (48\%).

From the physician perspective, the primary barrier to treatment among patients who declined MT was a lack of patient motivation; physicians reported that this was a barrier for, on average, $33 \%$ of their patients who declined MT (Table 4). This was followed by the patient not being well enough to receive treatment (26\%). Physicians' impression was that, on average, $26 \%$ of their patients who declined treatment needed or wanted a break from chemotherapy, and that those patients may reconsider treatment options at a later date. Residual toxicities and financial burden were 
Table 3 Challenges and motivators to choosing therapy among non-small cell lung cancer survey participants $(n=77)$

\begin{tabular}{|c|c|c|c|c|c|c|c|c|c|}
\hline \multirow[t]{3}{*}{ Challenges and motivators } & \multirow{2}{*}{\multicolumn{2}{|c|}{$\begin{array}{l}\text { Was a chal- } \\
\text { lenge or } \\
\text { motivator }\end{array}$}} & \multirow{3}{*}{$\begin{array}{l}\text { Not } \\
\text { reported } \\
(n)\end{array}$} & \multicolumn{6}{|c|}{ Level of influence ${ }^{\mathrm{a}}$} \\
\hline & & & & \multicolumn{2}{|c|}{$\begin{array}{l}\text { Very influential } \\
\text { for decision }\end{array}$} & \multicolumn{2}{|c|}{$\begin{array}{l}\text { Somewhat } \\
\text { influential for } \\
\text { decision }\end{array}$} & \multicolumn{2}{|c|}{$\begin{array}{l}\text { Influence } \\
\text { level not } \\
\text { reported }\end{array}$} \\
\hline & $n$ & $\%$ & & $n$ & $\%$ & $n$ & $\%$ & $n$ & $\%$ \\
\hline \multicolumn{10}{|l|}{ Challenges } \\
\hline Lack of information on MT & 12 & 15.6 & 1 & 2 & 16.7 & 6 & 50.0 & 4 & 33.3 \\
\hline Concern that MT will not be able to shrink existing tumor & 15 & 19.5 & 2 & 2 & 13.3 & 13 & 86.7 & 0 & 0.0 \\
\hline Concern that MT will not be able to extend life & 14 & 18.4 & 2 & 4 & 28.6 & 9 & 64.3 & 1 & 7.1 \\
\hline Duration of MT & 13 & 16.9 & 2 & 5 & 35.7 & 8 & 57.1 & 1 & 7.1 \\
\hline Frequency of MT & 13 & 16.9 & 3 & 4 & 26.7 & 10 & 66.7 & 1 & 6.7 \\
\hline $\begin{array}{l}\text { Non-medical costs associated with MT (e.g., childcare, } \\
\text { transportation) }\end{array}$ & 12 & 15.6 & 2 & 2 & 16.7 & 8 & 66.7 & 2 & 16.7 \\
\hline Medical costs associated with MT & 14 & 18.2 & 1 & 5 & 33.3 & 9 & 60.0 & 1 & 6.7 \\
\hline Distance to treatment center & 7 & 9.3 & 2 & 2 & 28.6 & 5 & 71.4 & 0 & 0.0 \\
\hline Severity of adverse effects of MT & 26 & 33.8 & 3 & 3 & 12.0 & 15 & 60.0 & 8 & 30.8 \\
\hline Impact on quality of life & 20 & 26.0 & 1 & 4 & 20.0 & 16 & 80.0 & 0 & 0.0 \\
\hline Lack of support from family and friends & 6 & 7.8 & 1 & 2 & 33.3 & 3 & 50.0 & 1 & 16.7 \\
\hline Lack of support from oncologist & 4 & 5.2 & 0 & 2 & 25.0 & 2 & 25.0 & 4 & 50.0 \\
\hline Obligations to family (spouse, partner, dependents) & 7 & 9.1 & 1 & 2 & 28.6 & 5 & 71.4 & 0 & 0.0 \\
\hline Other & 8 & 10.4 & & & & & & & \\
\hline \multicolumn{10}{|l|}{ Motivators } \\
\hline Desire to control lung cancer & 72 & 93.5 & 0 & 60 & 83.3 & 10 & 13.9 & 1 & 1.4 \\
\hline Desire to be proactive against lung cancer & 74 & 96.1 & 2 & 60 & 81.1 & 12 & 16.2 & 1 & 1.4 \\
\hline MT's ability to stop tumor growth & 72 & 93.5 & 0 & 62 & 86.1 & 9 & 12.5 & 0 & 0.0 \\
\hline MT's ability to extend life & 75 & 97.4 & 0 & 62 & 82.7 & 11 & 14.7 & 1 & 1.3 \\
\hline Obligations to family (spouse, partner, dependents) & 66 & 85.7 & 0 & 55 & 83.3 & 9 & 13.6 & 1 & 1.5 \\
\hline Desire to follow oncologist's treatment recommendations & 72 & 93.5 & 0 & 55 & 76.4 & 13 & 18.1 & 3 & 4.2 \\
\hline Fear of death & 45 & 58.4 & 0 & 35 & 77.8 & 10 & 22.2 & 0 & 0.0 \\
\hline Support of family and friends & 70 & 90.9 & 0 & 49 & 70.0 & 20 & 28.6 & 0 & 0.0 \\
\hline Support of oncologist & 72 & 93.5 & 0 & 54 & 75.0 & 17 & 23.6 & 0 & 0.0 \\
\hline Other & 22 & 28.6 & & & & & & & \\
\hline
\end{tabular}

$M T$ maintenance therapy

${ }^{a}$ Level of influence of each motivator and challenge was among those that indicated the motivator and challenge was present

Table 4 Physician-perceived treatment barriers for stage IIIb and stage IV non-squamous non-small cell lung cancer patients who decline therapy

\begin{tabular}{lcccc}
\hline Reason for not initiating maintenance therapy & \multicolumn{3}{c}{ Oncologists $(n=100)$ [\% of cases] } \\
\cline { 2 - 5 } & Mean & SD & Median & Range \\
\hline Patient does not want to continue treatment & 33.3 & 24.9 & 25 & $0-100$ \\
Patient is not well enough to continue treatment & 26.4 & 17.6 & 25 & $0-75$ \\
Patient response to first line is not adequate & 21.2 & 17.9 & 20 & $0-100$ \\
Patient needs/wants a break and may start maintenance at a later date & 16.1 & 15.0 & 15 & $0-66$ \\
Patient is experiencing residual toxicities from induction treatment & 15.2 & 16.3 & 10 & $0-75$ \\
Financial burden & 12.2 & 14.1 & 10 & $0-60$ \\
Other & & & & \\
Patient decides against & 5.0 & NA & 5 & $5-5$ \\
\hline
\end{tabular}

Physicians were asked to indicate in what percentage of cases each of the listed factors plays a role when eligible non-small cell lung cancer patients decline maintenance therapy in their practice

$N A$ not applicable, $S D$ standard deviation 
considered by physicians to be minor factors (15 and 12\%, respectively).

In the DCE, MT was recommended for $75 \%$ of the total of 1200 hypothetical patient profiles. Nearly all (98\%) physicians recommended MT for at least one of the 12 profiles presented to them, and $26 \%$ of physicians recommended MT for all 12 profiles. Continuation MT was recommended more frequently than switch MT (46\% vs. $29 \%$ of profiles). When MT was not recommended, the most frequent alternative suggestion was for the patient to take a break from chemotherapy and reconsider later (55\%).

Physicians were significantly less likely to recommend MT for patients who had progressed than for a patient with stable disease $(p<0.01$; Table 5$)$. Increasing patient age also significantly decreased the likelihood of physicians recommending MT $(p<0.01)$. Similarly, physicians were less likely to recommend MT when the hypothetical patient lacked treatment motivation $(p=0.02)$, particularly if the patient was also inconvenienced by the treatment $(p<0.01)$. Physicians were also significantly less likely to recommend MT to a hypothetical patient with severe renal co-morbidities than to a patient with no co-morbidities $(p<0.01)$; however, other co-morbidity levels did not have a significant impact on MT recommendations. The attributes of first-line treatment tolerability and insurance status did not have a significant impact on physician treatment preferences. Physicians recommended MT the least frequently when the scenario included disease progression, followed by lack of motivation and inconvenience for the patient, and an age of 80 years or older or a severe renal co-morbidity $(57,63,67$, and $67 \%$ of physicians recommending MT, respectively; Electronic Supplementary Material Fig. 1).

\section{Discussion}

This study identified factors influencing patients' and physicians' decision-making regarding therapy for advanced NSCLC and provides insight into this decision-making process. Patients were knowledgeable regarding the goals of therapy and reported anticipated outcomes that were consistent with phase III trials. Nearly all patients reported involvement of their oncologist in the decision-making process.
Table 5 Results of discrete choice experiment multiple logistic regression model for attributes that affect the decision to treat stage IIIb and stage IV non-squamous non-small cell lung cancer patients with maintenance therapy

\begin{tabular}{|c|c|c|c|c|c|c|}
\hline \multirow[t]{3}{*}{ Attribute } & \multicolumn{6}{|c|}{ Total sample $($ oncologists $=100 ;$ scenarios $=1200)$} \\
\hline & \multicolumn{6}{|l|}{ Fixed effects } \\
\hline & Coefficient & SE & $z$ & $p>|z|$ & Lower CI & Upper CI \\
\hline \multicolumn{7}{|l|}{ Response to treatment (vs. stable response) } \\
\hline Complete response & -0.13 & 0.24 & -0.56 & 0.58 & -0.60 & 0.34 \\
\hline Partial response & 0.32 & 0.25 & 1.28 & 0.20 & -0.17 & 0.82 \\
\hline Progression & -1.75 & 0.23 & -7.63 & 0.00 & -2.20 & -1.30 \\
\hline \multicolumn{7}{|l|}{ First-line treatment tolerability (vs. no AEs) } \\
\hline One grade $1 / 2 \mathrm{AE}$ & 0.27 & 0.24 & 1.12 & 0.26 & -0.20 & 0.73 \\
\hline Two grade $1 / 2$ AEs & 0.05 & 0.23 & 0.20 & 0.84 & -0.40 & 0.49 \\
\hline One grade $3 / 4$ AEs & 0.29 & 0.24 & 1.25 & 0.21 & -0.17 & 0.75 \\
\hline \multicolumn{7}{|l|}{ Age } \\
\hline Per 1-year increase in age & -0.03 & 0.01 & -5.18 & 0.00 & -0.05 & -0.02 \\
\hline \multicolumn{7}{|l|}{ Co-morbidities (vs. no active co-morbidities) } \\
\hline Severe renal impairment, on dialysis & -0.97 & 0.24 & -4.09 & 0.00 & -1.43 & -0.50 \\
\hline Mild renal impairment and not on dialysis & -0.11 & 0.25 & -0.43 & 0.67 & -0.59 & 0.38 \\
\hline Other $^{\mathrm{a}}$ & -0.34 & 0.25 & -1.40 & 0.16 & -0.83 & 0.14 \\
\hline \multicolumn{7}{|c|}{ Motivation/convenience (vs. motivated and not inconvenienced) } \\
\hline Motivated but inconvenient & 0.00 & 0.25 & 0.01 & 0.99 & -0.50 & 0.50 \\
\hline Not motivated but convenient & -0.58 & 0.24 & -2.41 & 0.02 & -1.06 & -0.11 \\
\hline Not motivated and not convenient & -1.38 & 0.24 & -5.80 & 0.00 & -1.84 & -0.91 \\
\hline \multicolumn{7}{|l|}{ Insurance status (vs. no co-pay) } \\
\hline $5 \%$ co-pay & 0.09 & 0.24 & 0.38 & 0.70 & -0.37 & 0.55 \\
\hline $10 \%$ co-pay & -0.25 & 0.24 & -1.06 & 0.29 & -0.71 & 0.21 \\
\hline $20 \%$ co-pay & -0.22 & 0.24 & -0.94 & 0.35 & -0.68 & 0.24 \\
\hline
\end{tabular}

$A E s$ adverse events, $C I$ confidence interval, $S D$ standard deviation, $S E$ standard error

${ }^{a}$ Other co-morbidities include hepatitis B, diabetes, chronic obstructive pulmonary disease, cerebrovascular disease, or cardiovascular disease 
Our study used decision-making related to MT as an example for treatment decision-making in advanced NSCLC. While the treatment landscape for NSCLC is changing very rapidly with the introduction of checkpoint inhibitors, the decision to use MT is still relevant for patients without actionable tumor mutations following treatment with platinum-based chemotherapy either alone or in combination with checkpoint inhibitors. Patients and physicians continue to consider motivators, barriers such as adverse effect profiles and impact on quality of life, as well as patient and disease characteristics in making treatment-related decisions.

The motivators identified in the interviews for accepting therapy were all considered influential by the majority of patients in the survey, with the primary motivator being the potential survival benefits. This aligns with previous research where survival was observed to be a key decisionmaking factor for patients regarding oncology treatments [9-13]. In a lung cancer patient consultation organized by the US Food and Drug Administration (FDA), the majority of patients stated that prolonged survival was the main factor influencing therapy decisions. However, some patients considered quality of life to be more important than survival. Clinical, social, and demographic characteristics, such as stage of disease, age, symptoms, and family circumstances, were influential factors on patients' opinions in this consultation [9]. A recent DCE of treatment preferences among patients with lung or colon cancer reported that survival was more important to patients than the impact of treatment on their physical capacity, appearance, food intake/digestion, and clinic waiting time [10].

The perceived challenges appeared to be varied in comparison with the motivators, and were infrequently acknowledged as influential by patients. The primary challenge from the patient perspective was the possibility of adverse effects. However, specific to MT, it was interesting to note that patients in the qualitative interviews reported that the adverse effects associated with MT, while a barrier, were anticipated to be less severe relative to first-line treatment and, therefore, were also viewed as a motivator.

It is crucial to have an understanding of the physician's perspective along with the patient's, as the physician was reported to be a main source of information about therapy options and was almost always involved in the decisionmaking process, in some cases making the decision with only minor or no perceived involvement of the patient, as reported by participants in our patient interviews and survey. Furthermore, most patients reported having several discussions about treatment options with their physician before a decision was made, and the physician's recommendation and the information provided in those discussions may be highly influential on the patient's decision. The importance of physician interactions in treatment decision-making was also recently shown for early-stage NSCLC patients when deciding about surgery and radiotherapy [19]. In a crosssectional survey conducted after a decision was made, but before treatment, $85 \%$ of patients indicated that decisionmaking should ideally be shared between the patient and physician. In addition, patients reported that their families played an important role in decision-making, which is in accordance with the findings from our study.

Previous studies have identified response to first-line therapy, histology, physical functioning, and the presence of biomarkers as being influential to physicians' MT recommendations [12-18]. Our study also identified response to first-line treatment as a key factor for physicians; however, surprisingly and contrary to NCCN guidelines, disease progression was not always a deterrent to a MT recommendation, based on the hypothetical profiles presented to physicians. This potential to go against accepted treatment guidelines might warrant further exploration in future studies. Younger age, patient motivation, and absence of comorbidities were other patient factors that influenced physicians' treatment recommendations. Additionally, physicians reported using MT among patient populations not included in NCCN guidelines for MT at the time of the survey. A recent survey of oncologists in the USA also reported that oncologists recommended lung cancer treatments outside of current recommended treatment strategies; approximately half of surveyed oncologists indicated that they would recommend chemotherapy for patients with advanced lung cancer with an ECOG PS of 3 (with no pain) [19].

Among the patients in our study, motivation to take therapy was high, as shown by the fact that 76 of 77 survey participants decided to take MT. However, physicians indicated patient motivation as a main barrier to therapy based on their experience in daily practice, which may indicate that our patient survey population was more motivated and more likely to accept MT than the population of NSCLC patients eligible for MT seen by the physicians participating in our survey.

The patient survey was limited by the sample size. Due to the poor outcomes associated with advanced NSCLC, the majority of screened patients had disease progression or were enrolled in clinical trials in the hope of achieving better outcomes, making them ineligible for this study. The target sample size for the patient survey $(n=100)$ was therefore not achieved despite the amended protocol enabling the survey to be administered by mail. Another limitation was that nearly all surveyed patients had chosen to receive MT, perhaps resulting in an over-representation of motivators and an under-representation of barriers. Additionally, recall bias may have altered patient's recollection of barriers to MT. Sampling bias may have occurred because patients who receive MT come into the clinics more frequently, enabling easier recruitment by participating sites. Therefore, the results of the patient survey may be more generalizable 
to patients who decided to take MT, and less to those who declined MT; future research should identify and explore the perspectives of patients who declined MT. Furthermore, some aspects of the decision-making process were specific to MT, such as the expectation of a reduction of adverse effects from first-line therapy. These aspects are not generalizable to overall treatment decision-making in advanced NSCLC.

A key strength of this study was the use of multiple approaches, in the form of patient qualitative interviews and patient and provider surveys, which provide a broader understanding of the factors that may influence MT decision-making. The perspectives of both patients and physicians were investigated, as both play an important role in the treatment decision-making. The physician survey recruited physicians from a national panel, which resulted in variation in regional representation and practice type.

\section{Conclusion}

This study identified factors that influence the patient's decision to choose and the physician's decision to recommend MT for advanced NSCLC. These include challenges perceived by patients that may act as a barrier to choosing treatment, as well as factors that motivated patients or improved their experience, including the discussions and information leading to their decision. Together, these data provide a broader understanding of the real-world decisionmaking process for MT, and may help guide physicians in treatment-related discussions with their patients.

Acknowledgements The authors thank Jacob Devine (ICON plc) for his help with coding the patient interviews.

\section{Compliance with Ethical Standards}

Data Availability Statement The data that support the findings of this study are not available publicly; however, future collaborative research using the data may be possible upon reasonable request from the authors.

Funding This study was funded by Eli Lilly and Company.

Conflict of Interest Suzanne McMullen and Kerstin Müeller are employees of ICON plc, a contract research organization (CRO) that was contracted by Eli Lilly and Company (Lilly) to conduct this study. Sarah Goring was an employee of ICON at the time the study was conducted, and is currently working for ICON as an independent contractor. Catherine Muehlenbein, Himani Aggarwal, Ana B. Oton, and Katherine B. Winfree are employees of Lilly and own stock in the company. Lisa M. Hess and Yajun Zhu are Lilly employees. Edward S. Kim has received consulting fees and honoraria as well as support for travel to meetings from AstraZeneca, Celgene, Boehringer Ingelheim, and Lilly. Mohamed Mohamed has received honoraria for speaker engagements from Eli Lilly, BMS, Merck, Boehringer Ingelheim, AstraZeneca, and Genentech. David Waterhouse has served on or moder- ated advisory boards for AbbVie, Amgen, and AstraZeneca, has had paid consulting engagements with BMS and Clinical Trial Services (CTI), and has been paid as a speaker by Lilly, BMS, Celgene, and Genenech/Roche. Antoinette Wozniak has received research funding from Boehringer Ingelheim, as well as honoraria/consulting fees from Boehringer Ingelheim, AstraZeneca, Takeda, Coherus Biosciences, Hospira, HUYA Bioscience, and Beyond Spring Biopharmaceuticals. Benjamin Levy has received research funding from Celgene and Boehringer Ingelheim, and consulting fees from Eli Lilly, AstraZeneca, Celgene, Pfizer, Merck, Genentech, and Takeda. Jennifer L. Ersek declares no conflict of interest.

Ethical Approval and Informed Consent All procedures followed were in accordance with the ethical standards of the responsible ethics committee and with the Helsinki Declaration of 1975, as revised in 2008 (5). Informed consent was obtained from all patients for being included in the study. This study has been approved by the relevant ethics boards (physician survey: Western Institutional Review Board [WIRB]; chart review: WIRB, Marshfield Clinic Research Foundation Institutional Review Board [IRB], Chesapeake IRB, IRB of Beth Israel Medical Center, University of Southern California Health Sciences IRB; Qualitative interviews: Cone Health IRB; patient survey: WIRB, Chesapeake IRB, Cone Health IRB).

Author Contributions SM, LH, BL, MM, SG, KM, CM, HA, EZ, AO, and $\mathrm{KW}$ were involved in designing and planning the study and developing study materials. SM, KM, and SG were responsible for operationalization of the study. EK, BL, MM, DW, and JE collected data at their respective clinics. SG conducted the statistical analysis. SM and $\mathrm{KM}$ wrote the manuscript; all authors reviewed, provided critical input, and approved the final version.

Open Access This article is distributed under the terms of the Creative Commons Attribution-NonCommercial 4.0 International License (http://creativecommons.org/licenses/by-nc/4.0/), which permits any noncommercial use, distribution, and reproduction in any medium, provided you give appropriate credit to the original author(s) and the source, provide a link to the Creative Commons license, and indicate if changes were made.

\section{References}

1. American Cancer Society. Cancer facts and figures 2018. Atlanta: American Cancer Society; 2018.

2. Larsson ML, Ljung L, Johansson BB. Health-related quality of life in advanced non-small cell lung cancer: correlates and comparisons to normative data. Eur J Cancer Care. 2012;21:1365-2354

3. Iyer S, Taylor-Stokes G, Roughley A. Symptom burden and quality of life in advanced non-small cell lung cancer patients in France and Germany. Lung Cancer. 2013;81:288-93.

4. Paz-Ares LG, Dediu M, et al. PARAMOUNT: final overall survival results of the phase III study of maintenance pemetrexed versus placebo immediately after induction treatment with pemetrexed plus cisplatin for advanced nonsquamous non-small-cell lung cancer. J Clin Oncol. 2013;31:2895-902.

5. Gridelli C, Thomas M, et al. Final efficacy and safety results of pemetrexed continuation maintenance therapy in the elderly from the PARAMOUNT phase III study. J Thorac Oncol. 2014;9:991-7.

6. Belani CP, Brodowicz T, Ciuleanu TE, et al. Quality of life in patients with advanced non-small-cell lung cancer given 
maintenance treatment with pemetrexed versus placebo (H3EMC-JMEN): results from a randomised, double-blind, phase 3 study. Lancet Oncol. 2012;13:292-9.

7. NCCN. NCCN guidelines, version 4. Non-small cell lung cancer. Washington: NCCN; 2015.

8. Joffe H, Yardley L. Content and thematic analysis. In: Marks D, Yardley L, editors. Research methods for clinical and health psychology. London: SAGE; 2004. p. 55-68.

9. Center for Drug Evaluation and Research, Center for Biologics Evaluation and Research. The voice of the patient: lung cancer. Center for Drug Evaluation and Research (CDER) and Center for Biologics Evaluation and Research (CBER); 2013. http:// wayback.archiveit.org/7993/20171114201750, https://www.fda. gov/downloads/ForIndustry/UserFees/PrescriptionDrugUserFee/ UCM379698.pdf

10. Schmidt K, Damm K, Vogel A, et al. Therapy preferences of patients with lung and colon cancer: a discrete choice experiment. Patient Prefer Adherence. 2017;11:1647-56.

11. Brundage MD, Feldman-Stewart D, Cosby R, et al. Cancer patients' attitudes toward treatment options for advanced nonsmall cell lung cancer: implications for patient education and decision support. Patient Educ Couns. 2001;45:149-57.

12. Blais N, Corrales-Rodriguez L. Personalized choice of maintenance therapies in non-small-cell lung cancer. Curr Oncol. 2012;19:S64-72.
13. Leighl NB. Treatment paradigms for patients with metastatic nonsmall-cell lung cancer: first-, second-, and third-line. Curr Oncol. 2012;19:S52-8.

14. Blanco JA, Toste IS, Alvarez RF, et al. Age, comorbidity, treatment decision and prognosis in lung cancer. Age Ageing. 2008;37:715-8.

15. Molina JR, Yang P, Cassivi SD, et al. Non-small cell lung cancer: epidemiology, risk factors, treatment, and survivorship. Mayo Clin Proc. 2008;83:584-94.

16. Carnio S, Novello S, Mele T, et al. Extending survival of stage IV non-small cell lung cancer. Semin Oncol. 2014;41:69-92.

17. Stinchcombe TE, Socinski MA. Considerations for second-line therapy of non-small cell lung cancer. Oncologist. 2008;13(Suppl 1):28-36.

18. Gerber DE, Rasco DW, Le P, et al. Predictors and impact of second-line chemotherapy for advanced non-small cell lung cancer in the United States: real-world considerations for maintenance therapy. J Thorac Oncol. 2011;6:365-71.

19. Mokhles S, Nuyttens JJME, de Mol M, Aerts JGJV, Maat APWM, Birim Ö, et al. Treatment selection of early stage non-small cell lung cancer: the role of the patient in clinical decision making. BMC Cancer. 2018;18:79. 